\title{
Prevalence of trigger factors in migraine
}

\author{
Deepak Jain ${ }^{1}$, Reenu Choudhary ${ }^{2 *}$ \\ ${ }^{1}$ Associate Professor, ${ }^{2}$ Senior Resident, ${ }^{\mathbf{1} 2}$ Dept. of Neuroscience, Sawai Man Singh Medical College, Jaipur, Rajasthan, India
}

*Corresponding Author: Reenu Choudhary

Email: drreenu2711@gmail.com

\begin{abstract}
Background: Migraine is one of the most common types of headache. Many patients with migraine have precipitating factors known as trigger factors, recognition of which may lead to a better diagnostic, preventive and therapeutic approach.

Objective: To study the prevalence of various trigger factors in patients with migraine, and to analyse various socio-demographic factors with respect to trigger factors.

Materials and Methods: A total number of 500 patients of either sex fulfilling the ICHD3 diagnostic criteria for migraine aged more than 15 years attending Neurology Department of SMS Medical College, Jaipur, between January 2018 and February 2019 were studied. Patient enrolled in the study underwent a detailed structured interview and the information regarding the characteristics of headache including the time of onset, duration, frequency, character, site and associated features with due stress on the trigger or precipitating factors. Chi-square and t-test were performed to determine the statistical significance of the results.

Results: As per the analysis, $91 \%$ of the patients reported more than one trigger factor for headache. Bright sunlight (43\%), physical activity (43\%), noise (42\%), stressful events (41\%), tea/coffee deprivation (38\%), travelling (34\%), hunger (33\%), sleep (31\%), and fatigue $(29 \%)$ were some of the major trigger factors reported by the sample. People living in urban areas reported a higher number of trigger factor as compared to rural areas, which includes factors such as stress and fatigue. Sleep deprivation and physical stress were higher among rural areas.

Conclusion: The present study presents a general idea about trigger factors related to headache in the North-western part of India. Bright sunlight, physical activity, noise, stressful events, tea/coffee deprivation, travelling, hunger, sleep, and fatigue parameters were some of the major trigger factors reported by the sample. Moreover, people living in urban areas reported a higher number of trigger factor as compared to rural areas.Hence including these into the diagnostic criteria should be taken into consideration. Future studies should focus on the whole context that surrounds the trigger and the probable trigger synergy in headache precipitation, as these could help in the prevention and management of these disabling conditions.
\end{abstract}

Keywords: Migraine, Trigger factor, Headache.

\section{Introduction}

Migraine is a primary headache disorder characteristically having a unilateral location, pulsating quality, moderate or severe intensity, aggravation by routine physical activity and association with nausea and photophobia and phonophobia but with varied clinical presentations. ${ }^{1}$ The pathophysiology of migraine is still unclear. Triggers of an attack may initiate a depolarizing neuroelectric and metabolic event like the spreading depression. ${ }^{2}$ Thus, the factors altering neuronal hyperexcitability should be determined. Migraine sufferers report excessive sensitivity to light, sound, motion, odours, and other sensory stimuli in and between acute migraine attacks. 3,4

The trigger factors are the immediate antecedents' stimuli of the headache that precipitate or aggravate the headache. ${ }^{5,6}$ Underlying trigger factors of migraine differ between individuals and even between attacks in any given patient. The recognition of the precipitating factors of migraine is very important, because avoidance from these factors may lessen the frequency and the severity of the attacks. ${ }^{7-9}$ Stress, sunlight and noise are the most commonly reported trigger factors. ${ }^{10,11}$ Menstruation, environmental factors, sleep disturbances, fatigue, and nutrition, are also frequently mentioned. ${ }^{12-14}$ Besides these well-known factors, there are also some other triggers. Noise, sleep disturbances, weather and crying, are some of the other precipitating factors recently reported. ${ }^{15-18}$ Thus, the proposed study focused on studying the prevalence of various trigger factors in patients with migraine, and to analyse various sociodemographic factors with respect to trigger factors.

\section{Materials and Methods}

The study analyzed 500 patients of migraine of either sex aged more than 15 years attending Neurology Department of SMS Medical College, Jaipur, between January 2018 and February 2019 for their routine follow up. The diagnoses were made in accordance with the criteria of the International Classification of Headaches-3nd Edition Beta (ICHD-III beta). ${ }^{19}$ The characteristics of headache featuring its onset, frequency, duration and type of pain and other associated non-headache symptoms that is vertigo and cervical pain were noted. All subjects were questioned by the same doctor at the outdoor visit. Demographic characteristics of all subjects were recorded. Detailed physical, including ophthalmological examination and neurological examination, was done. Medical history included current and previous diseases, medications (particularly, analgesics, antidepressant and oral contraceptive drugs). Family history of migraine and other diseases, and consumption caffeine, tea and smoking habits were asked. Potential trigger factors for headache were questioned in all patients. A set of questions concerning the trigger factors were prepared, and the information about the trigger factors was collected with a structured interview. 
SPSS-25 was used for all the analysis. Specifically, frequency analysis, descriptive analysis, chi-square analysis and t-test were performed to test the significance of the results.

\section{Results}

\section{Demographic Analysis}

As per the demographic analysis, majority of the patients were female (70\%) aged between 25-34 years. Out of 500 patients, approx. $55 \%$ of the patients were married, and nearly $50 \%$ of the patients were employed. Near about 50 percent of the patients had competed high education or above. The average frequency of tea and coffee intake among the patients was 2 and 1.5 cup/day, respectively. The patients reported around 5 cigarettes per day. The average disorder duration was of 8.5 years, with approx. 5 attacks per month. On average, the attack duration was of approx. 11 hours with attack intensity of 7.04 .

\section{Comparing Headache Type}

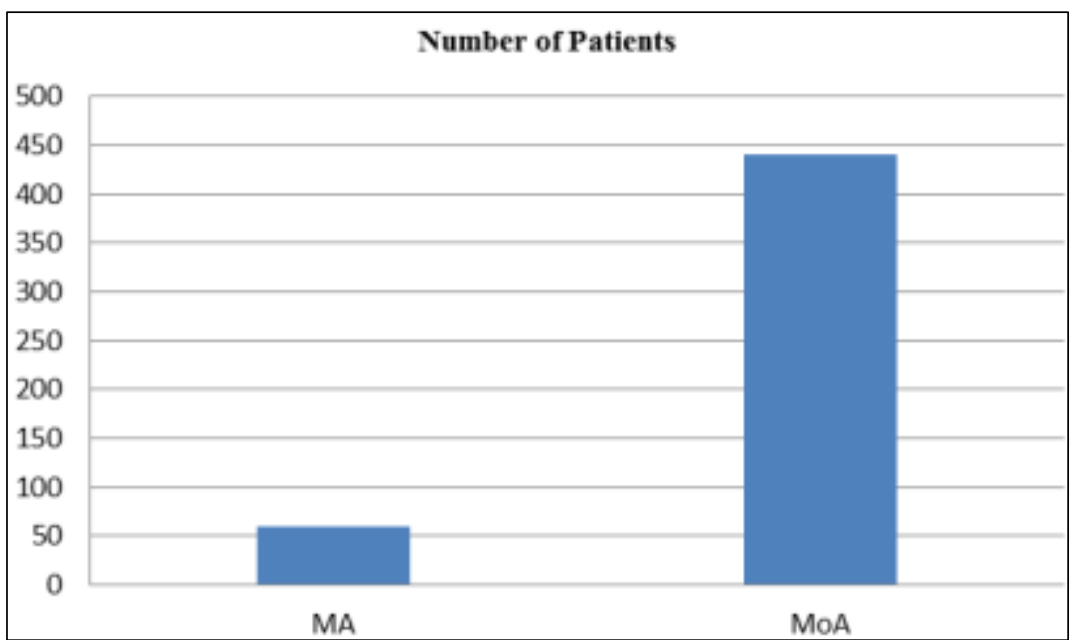

The table below shows a comparison between Migraine without Aura (MoA) and Migraine with Aura (MA) for different demographic parameters:

\begin{tabular}{|c|c|c|c|c|}
\hline & Total $(\mathrm{N}=\mathbf{5 0 0})$ & $\operatorname{MoA}(N=440)$ & MA $(\mathbf{N}=60)$ & p-value \\
\hline Men & 150 & 132 & 18 & \multirow{2}{*}{0.554} \\
\hline Women & 350 & 308 & 42 & \\
\hline $15-24$ & 113 & 103 & 10 & \multirow[t]{3}{*}{0.005} \\
\hline $25-34$ & 244 & 203 & 41 & \\
\hline $35-44$ & 143 & 134 & 9 & \\
\hline Married & 283 & 253 & 30 & \multirow[t]{2}{*}{0.331} \\
\hline Others & 217 & 187 & 30 & \\
\hline Without basic & 80 & 73 & 7 & \multirow[t]{5}{*}{0.024} \\
\hline Primary school & 89 & 80 & 9 & \\
\hline Secondary school & 104 & 93 & 11 & \\
\hline High school & 104 & 96 & 8 & \\
\hline Higher education & 123 & 98 & 25 & \\
\hline Employed & 243 & 212 & 31 & \multirow[t]{2}{*}{0.680} \\
\hline Unemployed & 257 & 228 & 29 & \\
\hline TEA-mean (cups/day) & 2.01 & 1.97 & 2.33 & 0.062 \\
\hline Coffee-mean (cups/day) & 1.39 & 1.40 & 1.37 & 0.851 \\
\hline Smoke - mean (cigarettes/day) & 4.85 & 4.76 & 3.051 & 0.057 \\
\hline Disorder duration-mean (years) & 8.53 & 8.56 & 8.32 & 0.670 \\
\hline Attacks frequency-mean (number/month) & 5.41 & 5.44 & 5.22 & 0.481 \\
\hline Attack duration-mean $(\mathrm{h})$ & 11.28 & 11.34 & 10.85 & 0.601 \\
\hline Attack intensity-mean $(1-10)$ & 7.04 & 7.00 & 7.27 & 0.172 \\
\hline Disability for work-mean $(1-3)$ & 1.92 & 1.93 & 1.88 & 0.706 \\
\hline Disability for everyday activities - mean (1-3) & 2.01 & 2.02 & 1.97 & 0.635 \\
\hline
\end{tabular}


As per the t-test and chi-square test performed, there is a statistically significant interaction between headache type and age $(\mathrm{p}<0.05)$. That is, people aged between 25-34 years reported MoA more as compared to MA. Similarly, there is a statistically significant interaction between headache type and education $(\mathrm{p}<0.05)$. That is, people with high or higher education reported MoA more as compared to MA. Apart from these two parameters, there is no statistically significant interaction between any of the other factor and headache types.

\section{Comparison of trigger factors between genders}

\begin{tabular}{|c|c|c|c|c|c|}
\hline \multirow[t]{2}{*}{ Trigger factors } & \multicolumn{2}{|c|}{ Total } & \multicolumn{2}{|c|}{ Gender } & \multirow[t]{2}{*}{ p-value } \\
\hline & $\mathbf{N}$ & $\%$ & Women & Men & \\
\hline Bright sunlight & 194 & 43 & 126 & 68 & 0.922 \\
\hline Physical activity & 194 & 43 & 112 & 82 & 0.815 \\
\hline Noises & 189 & 42 & 138 & 51 & 0.846 \\
\hline Stressful Event & 185 & 41 & 142 & 43 & 0.234 \\
\hline Tea deprivation & 171 & 38 & 112 & 59 & 0.496 \\
\hline Coffee deprivation & 153 & 34 & 99 & 54 & 0.56 \\
\hline Travelling & 153 & 34 & 87 & 66 & 0.626 \\
\hline Hunger & 149 & 33 & 114 & 35 & 0.77 \\
\hline Sleep deprivation & 140 & 31 & 80 & 60 & 0.143 \\
\hline Fatigue & 131 & 29 & 98 & 33 & 0.77 \\
\hline Cigarette smoke & 117 & 26 & 26 & 91 & 0.164 \\
\hline Air pollution & 117 & 26 & 59 & 58 & 0.722 \\
\hline Odors & 99 & 22 & 68 & 31 & 0.77 \\
\hline Menstruation & 98 & 28 & 98 & NA & NA \\
\hline Coffee consumption & 95 & 21 & 48 & 47 & 0.969 \\
\hline Studying & 90 & 20 & 51 & 39 & 0.431 \\
\hline Weather changes & 81 & 18 & 51 & 30 & 0.284 \\
\hline Cold drinks/ice cream & 63 & 14 & 23 & 40 & 0.953 \\
\hline Watching TV/Computer & 59 & 13 & 34 & 25 & 0.38 \\
\hline Premenstrual period & 49 & 14 & 49 & 0 & NA \\
\hline Chocolate & 32 & 7 & 18 & 14 & 0.541 \\
\hline Rain & 27 & 6 & 19 & 8 & 0.435 \\
\hline Fatty meals & 27 & 6 & 14 & 13 & 0.558 \\
\hline High altitude & 27 & 6 & 16 & 11 & 0.826 \\
\hline Dairy products & 23 & 5 & 13 & 10 & 0.104 \\
\hline Sexual activity & 14 & 3 & 9 & 5 & 0.697 \\
\hline
\end{tabular}

Chi-square analysis was performed between trigger factors and gender to determine if there is any statistically significant interaction between them. The results show that none of the trigger factors has statistically significant interaction with gender.

\section{Comparison of trigger factors among MoA and MA}

\begin{tabular}{|c|c|c|c|c|c|}
\hline \multirow{2}{*}{ Trigger factors } & \multicolumn{2}{|c|}{ Total } & \multicolumn{2}{c|}{ Headache Type } & \multirow{2}{*}{ p-value } \\
\cline { 2 - 5 } & $\mathbf{N}$ & $\mathbf{\%}$ & MoA & MA & \\
\hline Bright sunlight & 194 & 43 & 155 & 39 & 0.769 \\
\hline Physical activity & 194 & 43 & 151 & 43 & 0.68 \\
\hline Noises & 189 & 42 & 149 & 40 & 0.53 \\
\hline Stressful Event & 185 & 41 & 145 & 40 & 0.583 \\
\hline Tea deprivation & 171 & 38 & 140 & 31 & 0.93 \\
\hline Coffee deprivation & 153 & 34 & 125 & 28 & 0.337 \\
\hline Travelling & 153 & 34 & 122 & 31 & 0.272 \\
\hline Hunger & 149 & 33 & 121 & 28 & 0.879 \\
\hline Sleep deprivation & 140 & 31 & 115 & 25 & 0.583 \\
\hline Fatigue & 131 & 29 & 110 & 21 & 0.845 \\
\hline Cigarette smoke & 117 & 26 & 99 & 18 & 0.125 \\
\hline
\end{tabular}




\begin{tabular}{|c|c|c|c|c|c|}
\hline Air pollution & 117 & 26 & 96 & 21 & 0.492 \\
\hline Odors & 99 & 22 & 84 & 15 & 0.765 \\
\hline Menstruation & 98 & 28 & 80 & 18 & 0.335 \\
\hline Coffee consumption & 95 & 21 & 77 & 18 & 0.216 \\
\hline Studying & 90 & 20 & 73 & 17 & 0.048 \\
\hline Weather changes & 81 & 18 & 68 & 13 & 0.298 \\
\hline Cold drinks/ice cream & 63 & 14 & 49 & 14 & 0.681 \\
\hline Watching TV/Computer & 59 & 13 & 46 & 13 & 0.171 \\
\hline Premenstrual period & 49 & 14 & 40 & 9 & 0.425 \\
\hline Chocolate & 32 & 7 & 24 & 8 & 0.783 \\
\hline Rain & 27 & 6 & 20 & 7 & 0.582 \\
\hline Fatty meals & 27 & 6 & 19 & 8 & 0.68 \\
\hline High altitude & 27 & 6 & 18 & 9 & 0.891 \\
\hline Dairy products & 23 & 5 & 17 & 6 & 0.89 \\
\hline Sexual activity & 14 & 3 & 10 & 4 & 0.697 \\
\hline
\end{tabular}

Chi-square analysis was performed between trigger factors and headache to determine if there is any statistically significant interaction between them. The results show that none of the trigger factors has statistically significant interaction with headache types.

Trigger factors adjusted for sociodemographic data, anxiety and depressive symptoms

Neither MA nor MoA found to be statistically significantly associated with more triggers after being adjusted for sociodemographic data and anxiety/depressive symptoms [odds ratio (OR) 0.20 (95\% CI 0.09-0.31)]. Similarly, none of the trigger factors seems to be affected by these factors.

\section{Discussion \\ Prevalence of Headache Trigger Factors}

As per the results of the study performed, it has been found that around $91 \%$ of the patients had given at least one positive response towards the predicted list of trigger factor. The responses from the patients show that many of the patients reported more than 1 triggers. In both the study group, that is, MA and MoA, $87 \%$ of the patients reported at least two or more than two triggers. This result is in line with the migraine results of Kelman ${ }^{20}(94.6 \%)$, Fukui et al. ${ }^{21}$ (95.5\%), and Baldacci et al. ${ }^{22}(100.0 \%)$. Another major finding of the study was that attacks were precipitated incidentally by $73 \%$, whereas constantly only by $27 \%$. Thus, our findings support the previous studies, which also reported occasional triggers-induced pattern. Thus, it can be assumed that, firstly, the potential factors may not act alone, but various factors interact in combination with each other and, thus, increasing the likelihood of triggering a specific attack. Secondly, the potential factors may have different duration and intensity threshold for headache activation. And, thirdly, the potential factors may correlate with patients' natural history of headache with the consequent change in their occurrence.

\section{Trigger Factors Comparison among Headache Types}

The findings of the present study contradict the findings of Zivadinovet al. ${ }^{23}$ that subjects with MA report a higher median number of possible trigger per patient as compared to the MoA group. Our findings reveal that subjects with MoA report a higher median number of possible trigger per patient as compared to MA. Although, the reports no statistically significant interaction between any of the trigger factors and headache type, sleep, hormonal variations in women, stress and specific environmental triggers seemed to play a more important role in MoA subgroup.

\section{Most Important Trigger Factors Analysis Stressful Events}

Till date, the majority of the study on headache concluded that stressful events are one of the major trigger factors of a headache attack. The findings of the present study also support the earlier findings that stressful life events are the most common trigger in the whole sample, in both genders and among the two headache subtypes. There is a complex relationship between stress and headache crisis. It has been proposed that acute stress may affect the biological modulatory pathways leading to increased sensitivity of the migraine cortex. However, this is yet to be proved experimentally. The reason behind stress acting as the significant trigger can be explained by the findings of Houleet al. ${ }^{24}$ who detected a cumulative effect of combined high-stress levels and low sleep duration that influences the headache activity, thus, suggesting that stress acts as an enhanced trigger when it interacts with another factor.

\section{Female Harmonal Variance}

As per the study performed by Woberet al..$^{25}$, menstruation is the most important risk factor for the onset and the persistence of headache and migraine attack. In the present study, hormonal triggers like menstrual and premenstrual period were reported by $28 \%$ and $14 \%$ of the female patients, respectively. These results are similar to the previous study. Several studies have analysed the substantial mechanism between headaches and menses, and it has been suggested that that the fluctuation of serum estradiol and progesterone levels in the menstrual cycle is associated with higher headache activity in female migraineurs. 


\section{Sleep}

In the present study, $31 \%$ of the sample reported sleep deprivation as an important initiating factor for headache. According to Ho, changes in sleep hours, including night shift work with irregular shifts between nocturnal and diurnal schedule, appeared to be a strong trigger. It shows that individuals who performed shift work had more often headaches. This finding is also supported by Kelmanet al. ${ }^{20}$, who concluded that short sleep leads to increased severity and frequency of migraine attacks.

\section{Fatigue}

Near about $29 \%$ of the sample of the present study reported fatigue or tiredness as an important trigger factor. Moreover, the study also reveals that there is no statistically significant difference between the two headache subtypes. The physical exhaustion as a headache trigger is questionable due to its common presence as a prodromal symptom of migraine. However, the frequent occurrence of fatigue indicates that its cautious management may contribute to more efficient headache prevention.

\section{Whether Parameters}

Around $18 \%$ of the sample in the present study reported weather changes as a trigger factor for headache, which is similar to the results obtained by Robbins ${ }^{26}$. However, this finding is less significant compared to the higher percentages of other studies. The main reason for differences in results is due to different regional climatic conditions.

\section{Conclusion}

The present study presents a general idea about trigger factors related to headache in the North-western part of India. The results of the study revealed that majority of the patients reported more than one trigger factor for headache. Further, it was found that bright sunlight (43\%), physical activity (43\%), noise (42\%), stressful events (41\%), tea/coffee deprivation (38\%), travelling (34\%), hunger $(33 \%)$, sleep $(31 \%)$, and fatigue $(29 \%)$ parameters were some of the major trigger factors reported by the sample. Moreover, people living in urban areas reported a higher number of trigger factor as compared to rural areas, which includes factors such as stress and fatigue. Sleep deprivation and physical stress were higher among rural areas. None of the earlier studies found tea deprivation as a major trigger factor, but the present study reveals it as to be one of the major trigger factors which do not differ between gender and geographical location. MoA predominantly demonstrated higher frequencies in almost all triggers. However, there was no statistically significant difference between MoA and MA. Furthermore, taking into consideration, such a strong association of migraine and trigger factor, it should even be included in the diagnostic criteria. Future studies should focus on the whole context that surrounds the trigger and the probable trigger synergy in headache precipitation, as these could help in diagnosis, prevention and management of these disabling conditions.

\section{Strengths and Limitations of the Study}

One of the major strengths of the present study was that all patients were interviewed by the same clinician, so similar intervention was performed of each patient. Further, it was ensured that none of the patients was receiving preventive medication, and the diagnoses were precisely based on the revised criteria of ICD-III Beta. However, there were some limitations also attached to the present study. Firstly, the sample size and the geographical area for the study was limited. This can affect the applicability and validity of the results. Another limitation of the study was that patients were asked a large number of trigger factors. So there are chances that patients may have over-reported or wrongly reported the trigger factor.

\section{Conflict of Interest: None}

\section{References}

1. Mateen FJ, Dua T, Steiner T, Saxena S. Headache disorders in developing countries: Research over the past decade. Cephalalgia 2008;28:1107-14.

2. Leonardi $\mathbf{M}$. The global burden of migraine: Measuring disability in headache disorders with WHO's Classification of Functioning, Disability and Health (ICF) J Headache Pain 2005;6:429-40.

3. Woldeamanuel YW, Andreou AP, Cowan RP. Prevalence of migraine headache and its weight on neurological burden in Africa: A 43-year systematic review and meta-analysis of community-based studies. J Neurol Sci 2014;342:1-15.

4. Gururaj G, Kulkarni GB, Rao GN, Subbakrishna DK, Stovner LJ, Steiner TJ. Prevalence and sociodemographic correlates of primary headache disorders: Results of a population-based survey from Bangalore, India. Indian J Public Health 2014;58:241-8.

5. Linde M. Attitudes and burden of disease among selfconsidered migraineurs: A nation-wide population-based survey in Sweden. Cephalalgia 2004;24:455-65.

6. Muñoz P, Castillo J, Pascual J. Quality of life in chronic daily headache: A study in a general population. Neurology 2002;58:1062-5.

7. Souza-e-Silva HR, Rocha-Filho PA. Headaches and academic performance in universitystudents: A cross-sectional study. Headache 2011;51:1493-502.

8. Carod-Artal FJ, Ezpeleta D, Martín-Barriga ML, Guerrero AL. Triggers, symptoms, and treatment in two populations of migraineurs in Brazil and Spain: a cross-cultural study. Cephalalgia 2011;31:391-400.

9. Martin PR. Behavioral management of migraine headache triggers: learning to cope with triggers. Curr Pain Headache Rep 2010;14:221-27.

10. Cousins G, Hijazze S, Van de Laar FA, Fahey T. Diagnostic accuracy of the ID Migraine:a systematic review and metaanalysis. Headache 2011;51(7):1140-48.

11. Ghorbani A, Abtahi SM, Fereidan-Esfahani M, Abtahi SH, Shemshaki H, Akbari M, et al. Prevalence and clinical characteristics of headache among medical students, Isfahan, Iran. J Res Med Sci 2013;18

12. Johnson H, Guhl G, Arora J, Walling A. Migraine in students of a US medical school. Fam Med 2014;46(8):615-19.

13. Sacco S, Ricci S, Degan D, Carolei A. Migraine in women:the role of hormones and their impact on vascular diseases. $J$ Headache Pain 2012;13(3):177-89.

14. Agarwal V, Chaurasia RN, Mishra VN, Joshi D, Misra S. Clinical profile of headache from a tertiary care centre in eastern India. Int J General Med Pharm 2013;2(3):9-14. 
15. Senthil C, Gunasekaran N. Clinical profile of patients with chronic headache in a tertiary care hospital. Int $\mathrm{J} A d v \mathrm{Med}$ 2016;3:721-6.

16. Guruswamy A, Swamy S, Kavitha BB. Clinical profile of migraine headache with special reference to trigger factors in medical college set UP. Int J Sci Res 2017;6(7):1-5.

17. Stovner LJ, Andree C. Prevalence of headache in Europe: A review for the Eurolight project. J Headache Pain 2010;11:289-99.

18. Burch RC, Loder S, Loder E, Smitherman TA. The prevalence and burden of migraine and severe headache in the United States: Updated statistics from government health surveillance studies. Headache 2015;55:21-34.

19. Ray BK, Paul N, Hazra A, Das S, Ghosal MK, Misra AK, Banerjee TK, Chaudhuri A, Das SK. Prevalence, burden, and risk factors of migraine: A community-based study from Eastern India. Neurology India 2017;65:1280-88.

20. Kelman L. The triggers or precipitants of the acute migraine attack. Cephalalgia 2007;27(5):394-402.

21. Fukui PT, Gonçalves TR, Strabelli CG, Lucchino NM, Matos FC, Santos JP et al, Trigger factors in migraine patients. Arq Neuropsiquiatr 2008;66(3A):494-9.
22. Baldacci F, Lucchesi C, Cafalli M, Poletti M, Ulivi M, Vedovello $\mathrm{M}$ et al, Migraine features in migraineurs with and without anxiety-depression symptoms: a hospital-based study. Clin Neurol Neurosurg 2015 May;132:74-8.

23. Zivadinov R, Willheim K, Sepic-Grahovac D, Jurjevic A, Bucuk M, Brnabic-Razmilic O et al, Migraine and tension-type headache in Croatia: a population-based survey of precipitating factors.Cephalalgia 2003;23(5):336-43

24. Houle TT, Turner DP Golding AN, Porter JAH, Martin VT, Penzien DB et al, Forecasting Individual Headache Attacks Using Perceived Stress: Development of a Multivariable Prediction Model for Persons With Episodic Migraine. Headache 2017;57(7):1041-50.

25. Wöber C1, Wöber-BingölC.Triggers of migraine and tensiontype headache. Handb Clin Neurol 2010;97:161-72.

26. Robbins L. Precipitating factors in migraine: a retrospective review of 494 patients. Headache 1994 Apr;34(4):214-6.

How to cite this article: Jain D, Choudhary R. Prevalence of trigger factors in migraine. India J Neurosci 2019;5(2):53-8. 